\title{
Voltammetric detection of hydrochlorothiazide at molybdenum oxide modified screen-printed electrodes
}

\author{
M.F. Khanfar ${ }^{1}$, E. S. M. Abu-Nameh ${ }^{2}$, A. T. Afaneh ${ }^{2}$, M. M. Saket ${ }^{1}$, A. Ahmad ${ }^{2}$, W. Faraj ${ }^{1}$, M. \\ $\mathrm{Khalil}^{1}$, H. Al Khotaba ${ }^{2}$, M. Al Bujog ${ }^{2}$ \\ ${ }^{1}$ Pharmaceutical and Chemical Engineering Department, School of Applied Medical Sciences, German Jordanian \\ University, P.O.Box 35247 Amman 11180, Jordan \\ ${ }^{2}$ Department of Chemistry, Faculty of Science, Al-Balqa Applied University, Al-Salt 19117, Jordan
}

Received February 15, 2018; Accepted March 21, 2019

Electrochemical oxidation of hydrochlorothiazide (HCT) was investigated both experimentally and theoretically to explore the connection between thermodynamics and the oxidation potential. In this work, screen printed carbon electrodes were modified with molybdenum oxide by means of voltammetry. The modified surfaces demonstrated significant sensitivity toward hydrochlorothiazide detection in $\mathrm{pH} 2.00$-buffered aqueous solutions. The employed method was validated and used for hydrochlorothiazide quantification in commercial drug products. In this work, we presented results of density functional calculations for the standard reduction potential of the HCT $(\mathrm{H})_{2} \mid \mathrm{HCT}$ couple in aqueous solution. After consideration of 20 density functionals with 4 different basis sets, it was found that the hybrid meta functionals provide the most accurate prediction of the reduction potential in comparison with the available experimental data. The reported reduction potential is underestimated with GGA and MGGA and is overestimated with hybrid and meta hybrid functionals. Our results highlighted the importance of including solute-solvent hydrogen bonding effects in the theoretical modeling of redox processes.

Keywords: Molybdenum oxide, Hydrochlorothiazide, Screen-printed electrodes, Electrochemical deposition, Density functional theory (DFT).

\section{INTRODUCTION}

Hydrochlorothiazide is chemically 6-chloro-1,1dioxo-3,4-dihydro-2H-1,2,4-benzothiadiazine-7-

sulfonamide. Hydrochlorothiazide is a diuretic drug used for treatment of high blood pressure. For high blood pressure, it is often recommended as a first line treatment. HCT is taken orally and may be combined with other blood pressure medications as a single pill to increase the effectiveness, and decrease the accumulation of fluid (edema). It works by blocking salt and fluid reabsorption from the urine in the kidneys, causing increased urine output (diuresis). Hydrochlorothiazide is used to treat excessive fluid accumulation and swelling (edema) of the body caused by heart failure, cirrhosis, chronic kidney failure, corticosteroid medications, and nephrotic syndrome [1,2]. In Jordan, pharmaceutical analysis is mainly carried out by means of photometry and chromatography, a little attention is usually paid to electrochemistry. Therefore, in this work, we focused on the electrochemical analysis of HCT.[3-5]

\section{EXPERIMENTAL}

The EN AW-6060 alloy was chosen for the experimental investigation. The alloy was delivered by "AlCu metali d.o.o." company, in peak-aged state (T6 temper). The chemical composition was determined by using an optical emission spectrometer "Belec Compact Port" and is presented in Table 1. Peak-aged state was removed and O-temper achieved by annealing at $550{ }^{\circ} \mathrm{C}$ for $6 \mathrm{~h}$ in the electric resistance furnace Heraeus K$1150 / 2$.

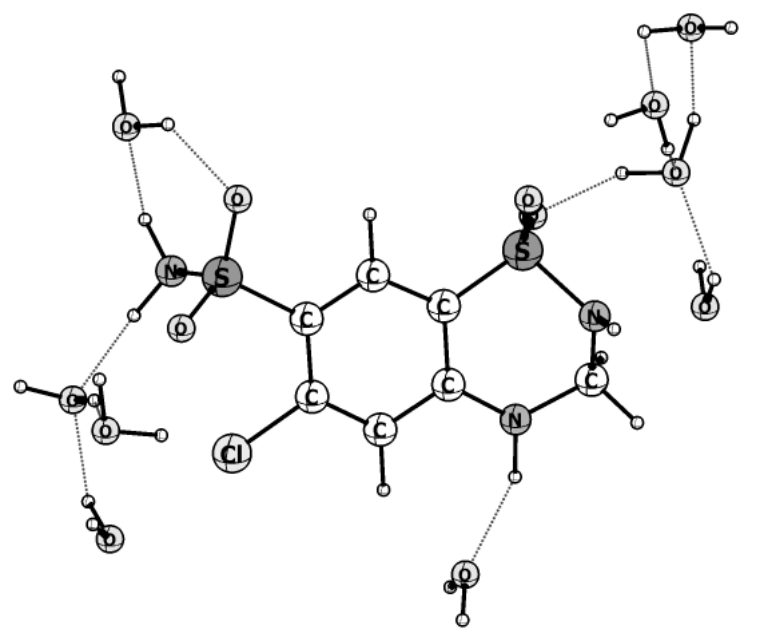

Figure 1. Structure of hydrochlorothiazide (HCT) with 9 water molecules.

Hydrochlorothiazide (HCT), Figure 1, is an active pharmaceutical of unique structural properties. It is electroactive (could be oxidized to its imine analogue), chromophore, due to the presence of a noticeable conjugated system, and as most of the active ingredients, it has an organic skeleton. With these properties, it could be quantified by a wide range of analytical methods

* To whom all correspondence should be sent: $\quad$ (C) 2019 Bulgarian Academy of Sciences, Union of Chemists in Bulgaria 
M.F. Khanfar et al.: Voltammetric detection of hydrochlorothiazide at molybdenum oxide modified ...

such as electrochemistry, spectroscopy, and chromatography [6-11].

Electrochemical methods are considered as the most convenient tools that could be used for HCT analysis, especially if the target analyte exists in the form of pharmaceutical dosage that is mainly blended with inactive, non-interfering species that may alter the detected signal attributed to HCT. These methods are simple, cheap, quick, and could be performed with portable hand-held equipment. By means of electrochemical methods, HCT has been analyzed using voltammetry (cyclic, differential pulse, square wave) and impedance spectroscopy, where the sensor is modified with a material which conductivity is affected by the quantity of HCT present in the analyte solution [1215].

Most of the sensors utilized for HCT are carbonbased materials. HCT has been analyzed on glassy carbon, multi-wall carbon nano tubes, graphite, graphene, and carbon paste electrodes. These carbon materials could be classified as unmodified, so that they are composed from carbonaceous material, or modified ones. Usually, carbon is blended with a modifier that elevates its conductivity so that the charge transfer rate across the electrode-electrolyte increases, or enhances the sensitivity so that the electrode becomes more responsive to the target analyte, especially at the low concentration levels. In the case of HCT, the carbon portion of the sensor has been mixed with conductivity enhancers such as ferrocene and its derivatives, or with sensitivity amplifiers such as metalloporphyrin [16-22].

One of the goals of modern quantum chemical simulations is to recognize and expect chemical reactivity, and another is to study the mechanisms of condensed-phase catalytic, photochemical and electrochemical reactions. Many reactions involve electron-transfer steps, sometimes combined with the breaking or formation of chemical bonds. Such reactions are significant in environmental chemistry, biochemistry, fuel cells, and electroanalytical field devices. The reduction potential is the key thermodynamic variable that describes the tendency of a chemical species to gain or lose electrons, which may be measured, for example, by cyclic voltammetry or spectrophotometric techniques combined with pulse radiolysis [23].

In this work, we report the experimental and theoretical studies of the oxidation of HCT compound. The experimental part combines both simplicity and detection sensitivity. Screen-printed carbon electrode (SPE) was utilized as the carbon foundation while molybdenum oxide was added as an electrochemical reaction catalyst. The SPE is an 308 almost $1 \mathrm{~cm}^{2}$ area sensor that confines the three electrodes needed for the voltammetric measurements, namely the working, the reference, and the counter electrode. With that configuration, aliquots of less than $500 \mu \mathrm{L}$ of the HCT solution could be analyzed for the active ingredient concentration. Molybdenum oxide has been electrochemically deposited on the carbon surface where molybdenum exits in the tetravalent oxidation state and hence acts as an electrondeficient center positive enough to oxidize the HCT to its imine counterpart.

The computational chemistry has evolved to the point that it is sometimes competitive to experiment to obtain precise values for certain molecular properties, density functional theory (DFT) [24-26]. In this study, the redox potential of hydrochlorothiazide was calculated with the employment of the density functional theory (DFT) molecular orbital calculations at a different level of theories (will be mentioned in detail in the computational methods section). The details of the calculation will be explained in the theoretical section of the present work.

\section{THEORETICAL CALCULATIONS}

The general procedure for computation of standard reduction potential $\mathrm{E}^{\circ}$ is given here. $\mathrm{E}^{\mathrm{o}}$ is related to the standard reduction free energy in solution given by

$$
E^{\circ}=-\frac{\Delta G_{s o l}^{D}}{n F}
$$

where $\mathrm{n}$ is the number of transferred electrons in the reaction which is equal to 2 for the reaction under investigation, $\mathrm{F}$ is the Faraday constant and $\Delta G_{s o l}^{o}$ is the free energy change associated with reduction at standard conditions. To calculate $\Delta G_{\text {sol }}^{\circ}$, it is necessary to use the following thermodynamic cycle (Born-Haber), which is used for transferring all of the species involved in the reaction from the gas to solution phase, Scheme 1.

The free energy change in the gas phase equals the adiabatic ionization potential, $\Delta G_{I}^{o}$, of the reduced species. Consequently, the aqueous standard-state reduction potential to the modified

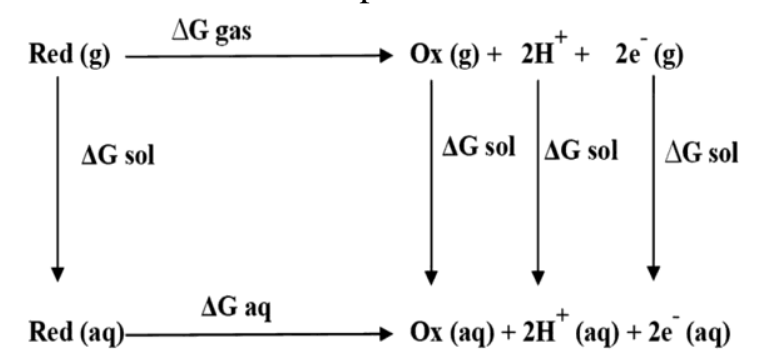

Scheme 1. Presentation of the 2-electron, 2-proton thermodynamic cycle 
M.F. Khanfar et al.: Voltammetric detection of hydrochlorothiazide at molybdenum oxide modified ...

electrode, $\Delta G_{M E}^{\circ}$ can then be estimated from equation (ii):

$$
E^{\circ}=\frac{\Delta G_{I}^{u}+\Delta \Delta G_{S}^{U}+\Delta G_{M E}^{\nu}}{n F}
$$

Finally, $\Delta \Delta \mathrm{G}_{\mathrm{S}}^{\circ}$ is the difference of solvation free energies of products and reactants,
$\Delta \Delta G_{\mathrm{S}}^{\circ}=\Delta G_{S}^{o}(O)-\Delta G_{S}^{o}(R)$.

\section{EXPERIMENTAL PART}

\section{Chemicals and reagents}

Britton-Robinson buffer (B-R buffer) with $\mathrm{pH}=$ 2.00 was prepared using $0.04 \mathrm{M} \mathrm{H}_{3} \mathrm{PO}_{4}, 0.04 \mathrm{M}$ $\mathrm{H}_{3} \mathrm{BO}_{3}$ (Honywell, U.S.A.), and $0.04 \mathrm{M} \mathrm{CH}_{3} \mathrm{COOH}$ (S-D Fine Chem. India). The desired $\mathrm{pH}$ value was obtained by the addition of the needed amounts of aqueous $0.2 \mathrm{M} \mathrm{NaOH}$ (VWR, U.K.) solution. Hydrochlorothiazide was a kind gift from the Jordanian Pharmaceutical Manufacturing Company. The pure hydrochlorothiazide (HCT) powder was used to prepare $2.22 \times 10^{-4} \mathrm{M}$ standard solution in the previously prepared B- $\mathrm{R}$ buffer $\mathrm{pH}=$ 2 solution. Standard solutions of HCT were prepared by serial dilution using the same buffer. Sodium molybdate (provided by Gainland Chemical Company, U.K.) and sodium sulfate (AZ Chemicals, China) were prepared as aqueous solutions with concentrations equal to $88.2 \mu \mathrm{M}$ and $3.22 \mathrm{mM}$, respectively.

\section{Instrumentation}

All of the voltammetric measurements were performed using PGSTAT 101 (Autolab, Metrohm, The Netherlands). Screen-printed electrodes (DRPC110) provided by Dropsense, Asturias, Spain, were used for the measurements. The electrodes have carbon working and counter electrodes and a silver reference electrode. Except for the molybdenum oxide deposition, unless otherwise stated, all of the voltammetric measurements are differential pulse with scan rate $=50 \mathrm{mV} / \mathrm{s}$, step potential $=5 \mathrm{mV}$, modulation amplitude $=25 \mathrm{mV}$, modulation time $=20 \mathrm{~Hz}$, interval time $=0.5 \mathrm{~s}$. The data were collected using the NOVA 2.0 software provided by Metrohm.

\section{Preparation of the modified electrodes}

The molybdenum oxide modified surface was prepared by the addition of $\mathrm{Na}_{2} \mathrm{MoO}_{4} / \mathrm{Na}_{2} \mathrm{SO}_{4}$ on the SPE and cycling potential of the working electrode between +0.20 to $-0.80 \mathrm{~V}$ at $50 \mathrm{mV} / \mathrm{s}$ for 10 cycles.

\section{Sample preparation}

The HCT content of two different commercial diuretic drugs was analyzed. Of each, ten tablets were weighed and powdered. A portion of the powder equivalent to the average weight of one tablet was transferred into a $25 \mathrm{ml}$ volumetric flask using about $15 \mathrm{ml}$ of $0.02 \mathrm{M} \mathrm{NaOH}$. The mixture was sonicated for $5 \mathrm{~min}$, then completed to volume with the same solvent. The resulted suspension was allowed to settle and $0.100 \mathrm{ml}$ from the supernatant was diluted to $25 \mathrm{ml}$ using the prepared B-R buffer of $\mathrm{pH} 2.00 .1 .00 \mathrm{ml}$ of the diluted solution was then diluted with $5.00 \mathrm{ml}$ of the buffer and $500 \mu \mathrm{L}$ of the solution was used for the voltammetric measurements.

\section{Computational methods}

The structure of HCT was optimized at different DFT/Basis sets level of theories in the gas phase. All the stationary points were characterized by frequency calculations at the same levels. The DFT we used in this study can be classified into four categories, specifically generalized gradient approximation (GGA), hybrid-GGA (H), metaGGA (MGGA), and hybrid meta-GGA (HM). Both GGA and MGGA exchange functionals depend on the electron spin density and its gradient; MGGA functionals also depend on the spin kinetic energy density. Both $\mathrm{H}$ and $\mathrm{HM}$ functionals depend on Hartree-Fock exchange, electron spin-density, and its gradient; HM functionals also depend on spin kinetic energy density. The GGA functionals considered here are BLYP, BP86, BPW91, BPBE and $\mathrm{PBE}$. The hybrid functionals that we used are B3LYP, B3P86, B3PW91, BH\&HLYP and PBEh. The MGGA functionals tested in the present work are BB95, mPWB95, PBEKCIS, TPSS and TPSSKCIS. The hybrid meta functionals that we considered here are B1B95, BB1K, MPW1B95, TPSS1KCIS and TPSSh [27,28].

The calculations were carried out with the Northwest Computational Chemistry Package (MWChem), version 6.3 [29].The choice of basis sets was made to be consistent with the valence basis set used for HCT compound: LANL2DZ, DZP (Dunning), cc-pVTZ and aug-cc-PVTZ [30,31].

The gas-phase calculations were followed with single points at the same level of theories in the aqueous environment. COSMO calculations at DFT/Basis sets levels were carried out on the stationary points to address solvation effects using the dielectric constant of water (78.39) in order to simulate the aqueous environment. In this study, we used the cluster model for the calculations of the solvation free energy for HCT. Put different, a hybrid solvation model was used in this study, implicit/explicit hybrid solvation model for the calculations. 
M.F. Khanfar et al.: Voltammetric detection of hydrochlorothiazide at molybdenum oxide modified ...

\section{RESULTS AND DISCUSSION}

\section{Modification of SPE by molybdenum oxide}

The deposition of molybdate ion on the SPE surface was accomplished by using a cycling potential of the working electrode between 200 and $-800 \mathrm{mV}$ versus reference electrode. The deposition process (shown in Figure 2) is an irreversible reduction of molybdate to molybdenum dioxide due to the absence of anodic features in the voltammogram. The reduction reaction is:

$$
\mathrm{MoO}_{4}{ }^{2-}{ }_{(\mathrm{aq})}+4 \mathrm{H}^{+} \text {(aq) }+2 \mathrm{e}^{-} \rightarrow \mathrm{MoO}_{2 \text { (ads) }}+2 \mathrm{H}_{2} \mathrm{O}_{(\mathrm{l})}
$$

The $\mathrm{pH}$ dependence of the reduction process is beyond the scope of the current study. The described cycling was repeated 10 times until a steady and stable voltammogram (not shown) was obtained.

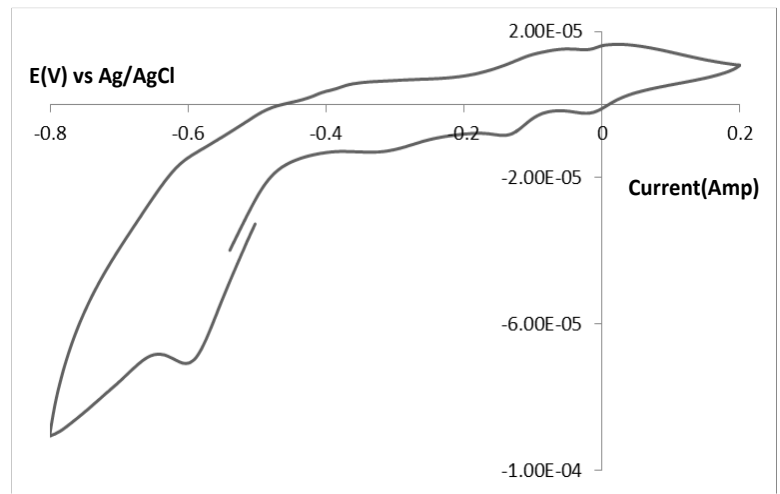

Figure 2. Deposition of molybdenum oxide on the SPE electrode. Cyclic voltammetry with scan rate $=50$ $\mathrm{mV} / \mathrm{s}$

\section{Oxidation of HCT}

The molybdenum-modified electrode was used for the oxidation of hydrochlorothiazide as shown in Figure 3. The modified surface demonstrates improved performance toward the oxidation reaction, when compared to its unmodified counterpart, appearing as enhancement of the oxidation current at $+0.950 \mathrm{~V}$.

Further research and investigation are needed to improve the reported catalytic activity that usually appears as a combination of the shift of the oxidation toward less positive potentials and enhancement in the oxidation to an extent higher than that reported in this work.

The oxidation of HCT is described in reaction (2) that shows how HCT undergoes two-electron, two-proton oxidation reaction and is converted to its imine counterpart:

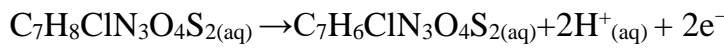

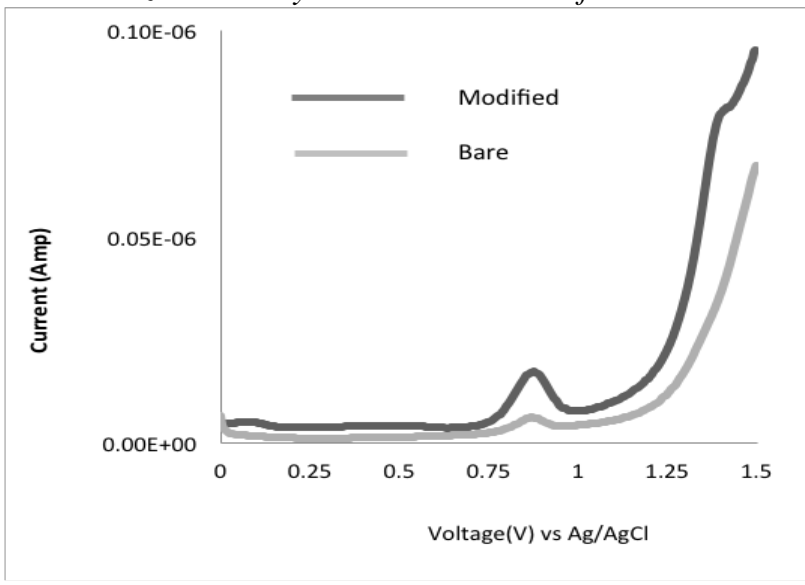

Figure 3. Effect of modification by molybdate on the oxidation of HCT

At potentials close to the value where the HCT oxidation occurs, molybdenum exists in the form of hydrogen molybdate that is reduced back to molybdenum dioxide previously deposited on the SPE surface [32]. The molybdenum oxide is generated as shown in reaction (3):

$\mathrm{HMoO}_{4(\mathrm{ads})}+3 \mathrm{H}^{+}{ }_{(\mathrm{aq})}+2 \mathrm{e}^{-} \rightarrow \mathrm{MoO}_{2(\mathrm{ads})}+2 \mathrm{H}_{2} \mathrm{O}_{(\mathrm{l})}$

Diffusion of the target analyte toward the electrode surface was studied by tracking the scan rate dependence of the oxidation peak current as shown in Figure 4.

According to Randless-Sevcik equation for diffusion-controlled electrochemical reactions, the peak electrolysis current depends on the square root of the employed scan. In this work, that dependence was examined over the $1-160 \mathrm{mVs}^{-1}$ scan range and linear adherence with $\mathrm{R}^{2}=0.9919$ was reported as shown in Figure 5 which confirms electrolysis of $\mathrm{HCT}$ at the modified surface.

\section{Validation of the analytical method}

In order to check the validity of the investigated method toward HCT in commercial tablets, four key analytical parameters were estimated for the employed method, namely, linearity, limit of detection, limit of quantitation, and recovery. By serial dilutions, the response of the modified electrode toward the oxidation of HCT was studied in the 1.42-29.77 ppm concentration range. 
M.F. Khanfar et al.: Voltammetric detection of hydrochlorothiazide at molybdenum oxide modified ...

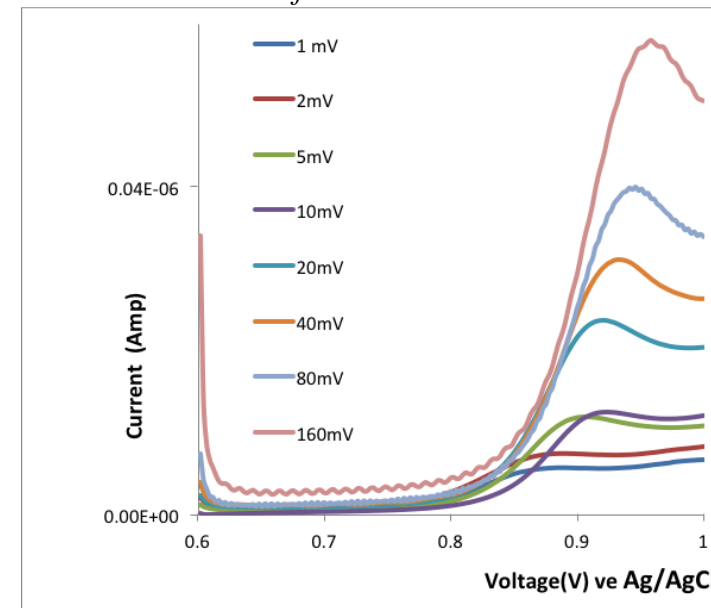

Figure 4. Dependence of the oxidation potential on the scan rate as obtained by linear sweep voltammetry.

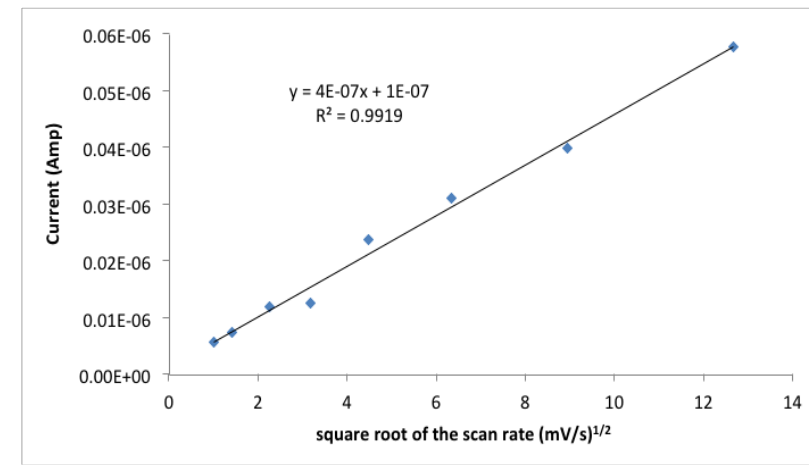

Figure 5. Dependence of the peak oxidation current on the scan rate.

The measurements were performed in sets of six trials per concentration in the mentioned range and the linear response of the modified SPE to elevation in the HCT concentration is shown in Figure 6. From the graph, the $\mathrm{R}^{2}$ value was found to be 0.9942 while the limits of detection and quantitation were calculated to be equal to 6.991 and $21.185 \mathrm{ppm}$, respectively.

Recovery was checked for two commercial drugs, namely monozide $(12.5 \mathrm{mg})$ and amuretic $(25 \mathrm{mg})$ and the corresponding recovery values were reported as 81.2 and $96.3 \%$, respectively. The relatively low recovery value reported for monozide could be attributed to the presence of species that may cause interference or masking.

\section{Standard reduction potential in DFT Model}

The resulting values of $\mathrm{E}_{\mathrm{HCT}(\mathrm{H})_{2} \mid \mathrm{HCT}}^{\circ}$ are given in

Table 1 for the calculations with 9 explicit water molecules with implicit solvation model, ISM, (hybrid model).

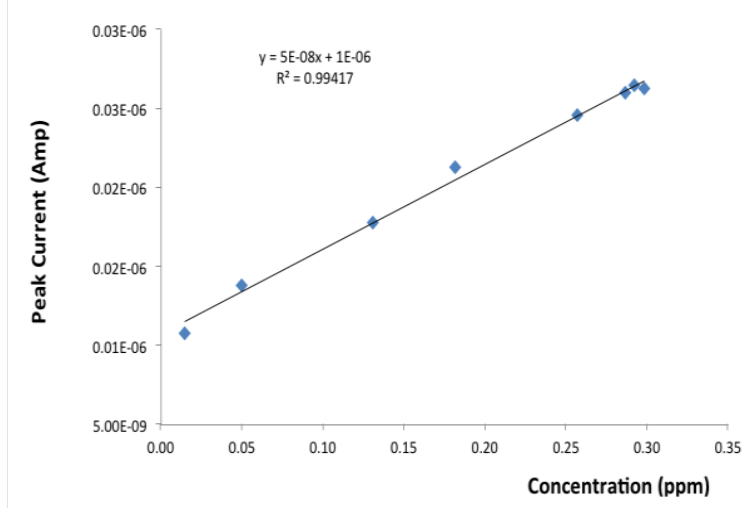

Figure 6. Calibration curve for the oxidation of HCT at the molybdenum-modified surface.

We also show the average values of $E^{o}$ and standard deviations of each combination of basis set and type of density functional. The values in Table 1 significantly differ from the values calculated with ISM. The corresponding values overestimated $E^{o}$ by over $1.62 \mathrm{~V}$ with all types of density functionals. The significant difference between standard reduction potentials computed using ISM and hybrid model stems from the different physical descriptions of the solvent. The hybrid model description contains many physical effects that are absent in the ISM, including solute flexibility, solute-solvent interaction, and hydrogen bonding. Furthermore, there is a probability that the errors in ISM are a consequence of incorrect parameterization of the atomic radii. Generally, the HM methods combined with aug-cc-pVTZ predict the experimental value of $\mathrm{E}_{\left.\mathrm{HCT}(\mathrm{H})_{2}\right] \mathrm{HCT}}^{\circ}=0.95 \mathrm{~V}$ better than the other methods. The GGA and MGGA predictions are underestimated with respect to the experiment, whereas the $\mathrm{H}$ and $\mathrm{HM}$ overestimate the corresponding values except for B3PW91/cc-pVTZ, PBEh/cc-pVTZ and PBEh/augccpVTZ hybrid GGA and MPW1B95/cc-pVTZ, TPSS1KCIS and TPSSh with all basis sets we used, Table 1.

Finally, using $\Delta G_{I}^{o}$ (MP2/aug-cc-pVTZ) along with the values of $\Delta \Delta G_{S}^{\circ}=-10.7$ and $-7.1 \mathrm{eV}$, respectively, for the 9 water molecules averaged over all the DFT results at the aug-cc-pVTZ basis level, we predict the following $\mathrm{E}_{\mathrm{HCT}(\mathrm{H})_{2} \mid \mathrm{HCT}}^{\circ}$ magnitude: $0.96 \mathrm{~V}$. Again, the inclusion of the explicit solvation shell in the treatment significantly improves the agreement of theory and experiment $(0.95 \mathrm{~V})$. 
M.F. Khanfar et al.: Voltammetric detection of hydrochlorothiazide at molybdenum oxide modified ...

Table 1. Standard reduction potential $\mathrm{E}_{\left.\mathrm{HCT}(\mathrm{H})_{2}\right] \mathrm{HCT}}^{\mathrm{O}}$ using ISM with hydration shell explicitly included.

\begin{tabular}{|c|c|c|c|c|c|c|c|c|c|}
\hline Method & LANL2DZ & $\begin{array}{l}\text { DZP } \\
\text { (Dunning) }\end{array}$ & $\begin{array}{l}\text { cc- } \\
\text { pVTZ }\end{array}$ & $\begin{array}{l}\text { aug-cc- } \\
\text { pVTZ }\end{array}$ & Method & LANL2DZ & $\begin{array}{l}\text { DZP } \\
\text { (Dunning) }\end{array}$ & $\begin{array}{l}\text { cc- } \\
\text { pVTZ }\end{array}$ & $\begin{array}{l}\text { aug- } \\
\text { cc- } \\
\text { pVTZ }\end{array}$ \\
\hline GGA & & & & & $\mathrm{H}$ & & & & \\
\hline BLYP & 0.81 & 0.86 & 0.70 & 0.83 & B3LYP & 1.17 & 1.22 & 1.00 & 1.10 \\
\hline BP86 & 0.89 & 0.90 & 0.75 & 0.88 & B3P86 & 1.61 & 1.63 & 1.45 & 1.52 \\
\hline BPW91 & 0.82 & 0.85 & 0.67 & 0.79 & B3PW91 & 1.07 & 1.09 & 0.87 & 1.00 \\
\hline BPBE & 0.79 & 0.82 & 0.64 & 0.75 & BH\&HLYP & 1.34 & 1.39 & 1.14 & 1.20 \\
\hline PBE & 0.79 & 0.81 & 0.63 & 0.74 & PBEh & 1.01 & 1.05 & 0.82 & 0.89 \\
\hline Average & 0.82 & 0.85 & 0.68 & 0.80 & Average & 1.24 & 1.28 & 1.06 & 1.14 \\
\hline SD & 0.04 & 0.04 & 0.05 & 0.06 & SD & 0.24 & 0.24 & 0.25 & 0.24 \\
\hline MGGA & & & & & $\mathrm{HM}$ & & & & \\
\hline BB95 & 0.79 & 0.84 & 0.67 & 0.80 & B1B95 & 1.11 & 1.15 & 0.87 & 0.90 \\
\hline mPWB95 & 0.82 & 0.87 & 0.71 & 0.81 & $\mathrm{BB} 1 \mathrm{~K}$ & 1.23 & 1.27 & 1.04 & 1.08 \\
\hline PBEKCIS & 0.85 & 0.87 & 0.69 & 0.80 & MPW1B95 & 1.15 & 1.18 & 0.89 & 1.02 \\
\hline TPSSKCIS & 0.75 & 0.80 & 0.62 & 0.72 & TPSS1KCIS & 0.88 & 0.90 & 0.72 & 0.82 \\
\hline TPSS & 0.70 & 0.77 & 0.59 & 0.67 & TPSSh & 0.84 & 0.85 & 0.67 & 0.75 \\
\hline Average & 0.78 & 0.83 & 0.66 & 0.76 & Average & 1.05 & 1.07 & 0.84 & 0.91 \\
\hline SD & 0.06 & 0.04 & 0.05 & 0.06 & SD & 0.17 & 0.18 & 0.15 & 0.14 \\
\hline
\end{tabular}

\section{CONCLUSIONS}

The analysis performed in this work shows that the modification of carbon electrodes with species that exist in different oxidation states enhances the voltammetric detection of target pharmaceuticals like HCT. The modified surface demonstrated promising performance toward the oxidation reaction; further studies could involve imaging of the modified surface and correlating topography of the modified surface to its activity.

Enhancement of the oxidation current in the presence of the adsorbed molybdates points to their catalytic activity; further effort in that direction may include modification of the screen printed electrodes with a binary adsorbate besides the molybdenum oxide.

We have presented the computational study of the aqueous reduction potential of the $\mathrm{E}_{\mathrm{HCT}(\mathrm{H})_{2} \mid \mathrm{HCT}}^{\circ}$ redox reaction. We used exchange-correlation functionals classified into four categories: GGA, MGGA, HGGA and HMGGA combined with four basis sets. The computational procedure is based on a thermodynamic cycle where the electrochemical parameters are determined by the ionization free energy, and the solvation free energy of the species. The reduction potential $\mathrm{E}_{\mathrm{HCT}(\mathrm{H})_{2} \mid \mathrm{HCT}}^{\circ}$ is overestimated when ISM is treated only whereas it is in good agreement with the experimental value when the explicit shell is included in the ISM.

The correlation between the experimentally reported reduction potential and that calculated theoretically is of significant importance, especially for the selection of the working electrode and the materials added to it.

\section{REFERENCES}

1 S.S. Badawy, B. AbdelAzeem, ChemXpress, 6, 14 (2014).

2 A.F. Alghamdi, J. Food Drug Analysis, 22, 363 (2014).

3 M.I.H. Helaleh, T. Korenaga, E.S.M. Abu-Nameh, R.M.A.Q. Jamhour, Pharmaceutica Acta Helvetiae, , 73, 255 (1999).

4 M.I.H. Helaleh, ESM Abu-Nameh, Anales de Química, 94, 160 (1998).

5 M.I.H. Helaleh, E.S.M. Abu-Nameh, R. Jamhour, Acta Poloniae Pharmaceutica, 55, 93 (1998).

6 S. S. Abd El-Hay, H. Hashem, A. A. Gouda, Acta Pharm., 66, 109 (2016).

7 H.W. Darwish, A. H. Bakheit, A.S. Abdelhameed, Acta Pharm. 66, 83 (2016).

8 K. Deventer, O.J. Pozo, P. Van Eenoo, F.T. Delbeke, J. Chromatogr. A, 1216, 2466 (2009).

9 L. Fei, X. Yu, S. Gaob, J. Zhang, Q. Guo, J. Pharm. Biomed. Analysis, 44, 1187 (2007).

10 R. Onnainty, E.M. Schenfeld, M.R. Longhi, M.A. Quevedo, G.E. Granero, Biomed. Chromatography, 31, 1 (2017). 
M.F. Khanfar et al.: Voltammetric detection of hydrochlorothiazide at molybdenum oxide modified ...

11 M.L. Jadhav, M.V. Girase, S.K. Tidme, M.S. Junagade, Int. J. Spectroscopy, 1 (2014).

12 A. Chmielewska, L. Konieczna, A. Plenis, H. Lamparczyk, J. Chromatogr. B, 839, 102 (2006).

13 M. Cristina, G. Santosa, C. Ricardo, T. Tarleya, L.H. Dall'Antonia, E.R. Sartoria, Sensors and Actuators B, 188, 263 (2013).

14 M.B. Gholivand, M. Khodadadian, Electroanalysis, 25, 51263 (2013).

15 N. Kumar, R.N. Goyalz, Melamine $/ \mathrm{Fe}_{3} \mathrm{O}_{4}$ Nanoparticles Based Molecular Imprinted Highly Sensitive Sensor for Determination of Hydrochlorothiazide: An Antihypertensive Drug, $J$. Electrochem. Soc., 164, B240 (2017).

16 B. Rezaei, S. Damiri, IEEE Sensors Journal, 8, 9, 1523 (2008).

17 B.S. Wesley, D. N. Machini, M. David-Parra, F.S. Teixeira, Materials Sci. Eng. C, 57, 344 (2015).

18 H. Beitollahi, F. Ghorbani, Ionics, 19, 1673 (2013).

19 E. Er, H. Çelikkan, M. L. Aksuc, N. Erka, Anal. Methods, 7, 9254 (2015).

20 O. Abdel Razak, J. Pharm. Biomed. Analysis, 34, 433 (2004).

21 H. Beitollahi, M. Hamzavi, M. Torkzadeh-Mahani, Materials Sci. Eng. C, 52, 297 (2015).

22 B. Uslu, S.1.A. Ozkan, Analytical Letters, 40, 817 (2007).
23 P. Wardman, Reduction J. Phys. Chem. Ref. Data, 18, 1637 (1989).

24 P. Hohenberg, W. Kohn, Phys. Rev. B, 136, 864 (1964).

25 M. Koch, C. Holthausen, A Chemists Guide to Density Functional Theory, Wiley-VCH, Weinheim, 2000.

26 R. G. Parr, W. Yang, Density Functional Theory of Atoms and Molecules, Oxford University Press, Oxford, 1989.

27 K. Burke, J. Chem. Phys. 136 (15), 150901 (2012).

28 A. D. Becke, J. Chem. Phys., 140 (18), 18A301 (2014).

29 M. Valiev, E. J. Bylaska, N. Govind, K. Kowalski, T. P. Straatsma, H. J. J. van Dam, D. Wang, J. Nieplocha, E. Apra, T. L. Windus, W. A. de Jong, Comput. Phys. Commun. 181, 1477 (2010).

30 D. Feller, J. Comp. Chem., 17, 1571 (1996).

31 K. L. Schuchardt, B. T. Didier, T. Elsethagen, L. Sun, V. Gurumoorthi, J. Chase, J. Li, T. L. Windus, Basis Set Exchange: A Community Database for Computational Sciences, J. Chem. Inf. Model., 47(3), 1045 (2007).

32 S. S. Viswanathan, L. Chi-Woo, ChemSusChem, 5, 1146 (2012). 